REVUE, BIBL,TOGRAPHIQUE

\title{
LA RACE OVINE ROMANOV
}

\author{
A. DESVIGNES $\left({ }^{1}\right)$ \\ avec la collaboration technique de Nathalie Boutler, Denise Boyajean et Nathalie Zuzine \\ Département de Génétique animale, \\ Centre national de Recherches zootechniques, I. N.R. A., \\ 78 - Jouy-en-Josas
}

\section{IN'TRODUC'TION}

Le facteur limitant d'une intensification de l'élevage ovin réside à l'heure actuelle dans le nombre d'agneaux commercialisés par brebis et par an. C'est pourquoi différents organismes nationaux de recherche, voire des firmes privées, s'intéressent à la sélection de races ou de souches de brebis susceptibles de donner un plus grand nombre d'agneaux soit par un niveau de prolificité élevé, soit par leur aptitude à réaliser deux agnelages par an.

Dès I962, l'Institut national de la Recherche agronomique a pris conscience de ce problème et a entrepris dans ce but une expérimentation dont nous aurons à reparler ultérieurement. L'amélioration de la productivité de nos races ovines à viande est envisagée conjointement par sélection en race pure et par des techniques de croisement avec des races réputées pour leur haut niveau de prolificité. Parmi ces dernières, figure la race Romanov, originaire d'U. R.S.S. et qui a fait l'objet dans ce pays de nombreuses publications d'aspect scientifique et technique.

Aussi nous proposons-nous d'exposer les caractéristiques zootechniques de cette race au cours de cette revue bibliographique. Après avoir situé son importance dans son pays d'origine, nous indiquerons successivement ses caractéristiques de reproduction, de croissance et de valeur bouchère, les méthodes préconisées pour son élevage et les expériences de croisement destinées à améliorer la prolificité d'autres races auxquelles elle a participé.

\section{I. - ORIGINE DE LA RACE ET DESCRIPTION}

En U. R.S. S., parmi les quelques quarante races d'un cheptel de près de 80 millions de brebis, la race Romanov est celle qui présente l'effectif le plus faible, de l'ordre de 815 ooo têtes (Kovnerev, I966), mais elle est certainement la plus originale de par l'utilisation qui en est faite et de par ses caractères exceptionnels concernant la reproduction. Elle a été décrite pour

(1) Adresse actuelle : Institut technique de l'Élevage ovin et caprin, 36, rue Fontaine, 75 - Paris $\left(9^{\mathrm{e}}\right)$. 
la première fois en 1802 par Plakhov, et son berceau d'origine semble être la province de Iaroslavl dont les principales villes sont Iaroslavl, Toutaïev (anciennement Romanov) et Ivanovo (LEBEDEv, 1958). Les avis diffèrent concernant son origine ethnique : certains pensent qu'elle serait issue d'un croisement entre une race locale et des béliers importés de Silésie en 1720 , ou de Hollande en 1754. (Sokolov, I 853, relate que Pierre Ier a fait venir dans la zone en I 716 deux bergers de Silésie qui auraient pu amener des béliers avec eux). Par contre, VorobIEv (I966) cite différentes études réalisées entre 1853 et 1935 tendant à montrer que cette race ne comportait aucun apport de sang étranger. Vraisemblablement obtenue par sélection au cours de nombreuses années à partir de la race nordique à queue courte (SEmentschenko, I952; Szymski, 1954), elle était connue depuis fort longtemps sur les riches pâturages des rives de la Volga et s'est étendue ensuite dans d'autres zones : Selianine (1960) rapporte qu'elle a été introduite vers $193^{\circ}$ dans les districts Ouraliens et Trans-Ouraliens (provinces de Sverdlovsk, Kirov, Perm, Tioumen et République d'Udmurt). Actuellement élevée dans plus de 30 régions d'U. R. S. S., les principales zones restent Iaroslavl et Ivanovo où le cheptel ovin est roo p. Ioo de race Romanov, Kostroma, Volodga, Arkangelsk et Kalinine avec 75 à 80 p. roo (MojaEva, I965).

KovNerev et al. (1967) donnent à cette race la description suivante : animal haut sur pattes, au corps en forme de tonneau, aux côtes rondes, à la tête petite et anguleuse, aux oreilles dressées et mobiles, aux yeux volumineux, à queue courte et effilée de $13 \mathrm{~cm}$ de longueur. Les mâles, cornus ou non, ont un chanfrein plus busqué que les femelles, et une tête plus large et plus courte. Ils indiquent différentes mensurations reportées au tableau I qui montrent l'augmentation de format entre 1914 et 1963 .

\section{TABLEAU I}

Mensurations d'animaux de 2 ans et plus (en $\mathrm{cm}$ ) (d'après KovNerev et al., 1967)

\begin{tabular}{|c|c|c|c|c|c|c|c|c|}
\hline & Année & $\begin{array}{l}\text { Hauteur } \\
\text { au garrot }\end{array}$ & $\begin{array}{l}\text { Longueur } \\
\text { oblique }\end{array}$ & $\begin{array}{l}\text { Tour } \\
\text { de poitrine }\end{array}$ & $\begin{array}{l}\text { Profondeur } \\
\text { de poitrine }\end{array}$ & $\begin{array}{c}\text { Largeur } \\
\text { de poitrail } \\
\text { derrière les } \\
\text { omoplates }\end{array}$ & $\begin{array}{l}\text { Longueur } \\
\mathrm{du} \text { bassin }\end{array}$ & $\begin{array}{l}\text { Longueur } \\
\text { de tête }\end{array}$ \\
\hline \multirow{2}{*}{ Mâles } & 1914 & 63,0 & $6^{\prime}+8$ & 79,1 & 30,0 & 15,0 & 21,2 & 23,0 \\
\hline & 1963 & 69,3 & 73,8 & 108,0 & 34,6 & 25,1 & 23,5 & 23,3 \\
\hline \multirow{2}{*}{ Femelles } & 1914 & 62,6 & 61,9 & 76,6 & 28,0 & 15,0 & - & - \\
\hline & 1963 & 66,3 & 72,2 & 93,4 & 34,3 & 23,1 & 22,9 & 22,6 \\
\hline
\end{tabular}

La toison, noire à la naissance, apparaît ensuite gris bleuté du fait d'un mélange de poils noirs et de laine blanche. Le rapport nombre de poils/nombre de fibres de laine varie suivant les animaux de $\mathrm{I} / 4$ à $\mathrm{I} / \mathrm{LO}$, la densité totale de fibres étant de l'ordre de 24 à 30 fibres par $\mathrm{mm}^{2}$.

Alors que les poils ont une longueur de 3 à $4 \mathrm{~cm}$ et un diamètre de 60 à go microns, les fibres de laine ont de 5 à $8 \mathrm{~cm}$ de long et un diamètre de 18 à 24 microns (finesse de laine comparables à celles des Mérinos). Il existe parfois des fibres hétérotypiques d'un diamètre de 35 à 45 microns qui sont défavorables au bouclage de la toison. Enfin, les mâles présentent une crinière de longs poils noirs autour du cou et sur le fanon, représentant une surface de 2 à $3 \mathrm{dm}^{2}$ à 9 mois, et $5 \mathrm{dm}^{2}$ chez les adultes. Le mélange de poils ct de laine assure des qualités d'isolation thermique et de résistance au tassement tout à fait particulières : le coefficient d'isolation est deux fois à deux fois et demie supérieur à celui des toisons de type Mérinos. Une pelisse, composée 
de poils de $3 \mathrm{~cm}$ de long et 82 microns, dans le rapport de $1 / 7$, conserve sous des pressions de $5 \mathrm{~kg} / \mathrm{m}^{2}$ et $50 \mathrm{~kg} / \mathrm{m}^{2}$, des hauteurs respectives de $2,8 \mathrm{~cm}$ et $\mathrm{I}, 6 \mathrm{~cm}$.

Par ailleurs, les qualités technologiques de la peau (tabl. 2) plus particulièrement sa finesse, sa résistance et son élasticité, font que la fourrure d'agneaux de 6 mois est particulièrement recherchée pour la confection de vêtements chauds et néanmoins légers. C'est pourquoi le Romanov est considéré en U. R. S. S. comme une race à deux fins : fourrure et viande.

\section{TABLEAU 2}

Caractéristiques de la peau de Romanov à différents âges

(d'après KovNerev et al., 1967)

\begin{tabular}{|c|c|c|c|}
\hline & Agneaux 6 mois & Agneaux 9 mois & Adultes $3-4$ ans \\
\hline \multirow{2}{*}{ Surface de peau (en $\left.\mathrm{dm}^{2}\right)\left\{\begin{array}{l}\text { brute } \ldots \ldots \ldots \\
\frac{\text { traitée } \ldots \ldots \ldots}{\ldots}\end{array}\right.$} & 63 & 74 & 99,2 \\
\hline & 58 & 68 & 94,0 \\
\hline \multirow{2}{*}{ 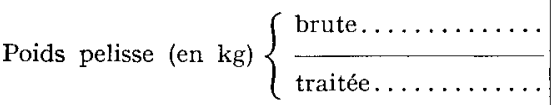 } & 1,8 & 2,2 & 2,36 \\
\hline & 0,6 & 0,9 & 1,16 \\
\hline Poids de $1 \mathrm{~m}^{2}$ pelisse traitée $\ldots \ldots \ldots \ldots$ & 1,05 & 1,3 & 1,24 \\
\hline Épaisseur de peau $(\mathrm{mm}) \ldots \ldots \ldots \ldots \ldots$ & 0,9 & 1,1 & 1,2 \\
\hline Résistance au déchirement $\left(\mathrm{kg} / \mathrm{mm}^{2}\right) \ldots$ & 1,6 & 2,1 & 2,7 \\
\hline Élasticité en pourcentage pour $0,5 \mathrm{~kg} / \mathrm{mm}^{2}$.. & 42,4 & 34,6 & 36,4 \\
\hline
\end{tabular}

\section{II. - CARACTÈRES DE REPRODUCTION}

\section{I. - Maturité sexuelle et âge à la première lutte}

Le Romanov se signale par une maturité sexuelle très précoce (DUкіNe, I966) puisque les mâles sont aptes à la lutte dès 3 à 4 mois et que certaines agnelles mettent bas à 9 mois. PANiNE (1957) pense qu'il s'agit là du résultat d'une sélection naturelle liée au fait que les mâles et les femelles étaient élevés en commun.

Sans toutefois préconiser la mise à la lutte des agnelles dès la puberté, les auteurs montrent qu'un agnelage relativement précoce n'est pas préjudiciable au développement ultérieur des femelles :

- Panine (I957) indique que des agnelles bien nourries peuvent atteindre le poids de $4^{\circ} \mathrm{kg}$ à 6 ou 8 mois, et être saillies à cet $\mathrm{a} g e$.

- Dobrotvorskaia (I965) mettant en lutte des agnelles âgées respectivement de IO-I I mois et de 18 mois obtient des performances d'agnelage et un développement à deux ans tout à fait semblables.

- Selianine (I965), comparant les performances de quatre groupes de brebis mises à la lutte à différents âges et selon différents niveaux d'alimentation (tabl. 3) conclut qu'une mise à la lutte des agnelles de 6-7 mois, alors que leur poids atteint seulement $56 \mathrm{p}$. Ioo du poids 
adulte, retarde leur croissance de I0,7 p. Ioo. Par contre, il recommande la mise en lutte à IoI I mois à condition d'assurer aux animaux une nourriture abondante.

TABLEAU 3

Influence de l'âge à la première lutte sur les performances de reproduction (SELIANINE, I965)

\begin{tabular}{|c|c|c|c|c|}
\hline & \multirow{2}{*}{$\begin{array}{l}\text { Lutte } \\
\text { à } 18 \text { mois }\end{array}$} & \multirow{2}{*}{$\begin{array}{l}\text { Lutte } \\
\text { à } 6-7 \text { mois }\end{array}$} & \multicolumn{2}{|c|}{ Lutte à 10-11 mois } \\
\hline & & & $\begin{array}{l}\text { Alimentation } \\
\text { poussée }\end{array}$ & $\begin{array}{l}\text { Alimentation } \\
\text { normale }\end{array}$ \\
\hline Nombre de femelles $\ldots \ldots \ldots \ldots$ & 280 & 64 & 26 & 31 \\
\hline Poids à la $1^{\text {re }}$ lutte $(\mathrm{kg}) \ldots \ldots$ & 35,6 & 23,6 & 33,3 & 28,4 \\
\hline Poids à la $1^{\text {re }}$ mise bas ....... & 38,1 & 25,6 & 36,0 & 30,1 \\
\hline Poids à la $2^{\mathrm{e}}$ mise bas $\ldots \ldots \ldots$ & 42,5 & 34,4 & 42,3 & 37,1 \\
\hline Prolificité au $1^{\text {er }}$ agnelage $\ldots$. & 1,70 & 1,41 & 1,68 & 1,60 \\
\hline Prolificité au $2^{\mathrm{e}}$ agnelage $\ldots$. & 1,90 & 1,71 & 1,94 & 1,78 \\
\hline Poids naissance des agneaux : & & & & \\
\hline 1er $^{\mathrm{er}}$ agnelage $\ldots \ldots \ldots \ldots$ & 2,8 & 2,2 & 2,7 & 2,5 \\
\hline $2^{e}$ agnelage $\ldots \ldots \ldots \ldots$ & 3,0 & 2,6 & 3,0 & 2,8 \\
\hline & & & & \\
\hline
\end{tabular}

\section{2. - Saison sexuelle et double agnelage}

On peut s'étonner de voir les auteurs réaliser des expériences de mise à la lutte d'agnelles à 6 , ro ou $\mathrm{r} 8$ mois sans se heurter aux problèmes de venue en chaleurs à certaines époques de l'année que nous connaissons chez nos races très saisonnées. En fait, la race Romanov présenterait cette particularité tout à fait remarquable de n'avoir pratiquement pas d'anœestrus saisonnier : dès I 802, Plakrov note l'aptitude des brebis à être saillies en toute saison, SmIRnov (1935), Ussakova et FudelJ (I94I) signalent la possibilité d'obtenir deux agnelages par an avec des luttes réalisées en juillet-août et en janvier-février. Il ne semble pas s'agir là d'observations de caractère exceptionnel puisque ces derniers auteurs indiquent que le double agnelage annuel a pu être réalisé sur des effectifs de I 540,925 et 724 brebis dans différents élevages. Par ailleurs, Kovnerev (1965) cite des élevages qui réalisent couramment cette performance sur 30 à 40 p. Ioo de leurs effectifs. Mais il préconise (KovNEREv et al., 1967) de ne remettre en lutte que les brebis ayant donné un ou deux agneaux lors du précédent agnelage.

Parallèlement à l'absence d'anœstrus saisonnier, les brebis reviennent en chaleur entre 30 et $4^{\circ}$ jours après la mise bas et peuvent être fécondées aussitôt (STrourov, I943; GUERASsimov, I957; Panine, I957; Pokrovskaik, I957; et Selianine, I960). De plus la durée de gestation étant réduite de plusieurs jours par rapport aux autres races, du fait d'une taille de portée élevée (Kovnerev et al., I967, observent des durées moyennes de I43-I44 jours avec des extrêmes de I39-I 49), on voit qu'il est possible de réaliser deux cycles de reproduction en 365 jours.

Il nous apparaît regrettable qu'aucun auteur n'ait étudié l'influence du double agnelage au cours de plusieurs années successives sur les performances et la longévité des brebis pour tester les possibilités d'utilisation intensive de cette race.

\section{3. - Prolificité (nombre d'agneaux nés par brebis agnelant)}

Sans citer tous les auteurs mettant en évidence la prolificité élevée de cette race, signalons que les valeurs moyennes observées s'étalent de I,84 à 3,20. Les performances individuelles les 
plus élevées sont citées par Smirnov (1935) et Pokrovskaia (1957) avec des portées de 7 à 9 agneaux, le record absolu semblant appartenir à une brebis ayant donné naissance à 64 agneaux en 12 mises bas.

Youdine et Petrov (1957) attribuent ceci au fait quautrefois seules étaient conservées pour la reproduction les agnelles provenant de portées triples ou quadruples. Mais plus récemment, l'abandon d'un tel critère de sélection a ramené dans beaucoup d'élevages le taux de prolificité à $\mathrm{I}, 97$, les meilleurs se situant toujours à 2,62 avec 245 agneaux sevrés pour Ioo brebis agnelant.

Notons que ces moyennes élevées ne sont généralement pas le fait de performances individuelles exceptionnelles: en I943, Stroukov note que sur 29 I 000 mises bas, i 8 p. Ioo sont simples, $47 \mathrm{p}$. I oo doubles et $35 \mathrm{p}$. I oo triples ou supérieures. Plus récemment (I967), KovNEREV et al., observent sur Ito 600 mises bas une moyenne de 2,15 agneaux pour respectivement 20,2 p. Ioo, 5I,3 p. Ioo et 24,2 p. Ioo de portées simples, doubles ou triples contre 4,3 p. Ioo de portées supérieures à 3 agneaux. Dans les élevages atteignant 2,5 à 2,6 agneaux, cette répartition est la suivante : 6 à 8 p. I0O, $3^{8}$ à 40 p. IOO, et 44 et $4^{6}$ p. IOO contre 8 à IO p. IOO.

On retrouve également cette homogénéité de production lors des agnelages successifs d'une même brebis : Sementschenko (I952) cite une brebis ayant donné 33 agneaux en 8 agnelages avec une portée maximum de 5 , et une autre ayant donné 2 I agneaux en 4 agnelages avec ure portée maximum de 6. Smirnov (1935) note que les femelles donnant I, 2 et 3 agneaux lors de leur premier agnelage donnent respectivement lors des agnelages ultérieurs I,89-2, I5 et 2,68 agneaux en moyenne. Le caractère de prolificité est donc répétable et il paraît égalemcnt être héritable : outre les observations de Smirnov (I935) portées au tableau 4, KovNEREV (I963) signale que les femelles nées simples ont un taux de prolificité inférieur de 24 à $\mathrm{I} 6 \mathrm{p}$. Ioo à celu $\mathrm{i}$ des brebis nées doubles, alors que les triples et les quadruples présentent respectivement une supériorité de $I_{7}$ à $30 \mathrm{p}$. Ioo et $\mathrm{I}, 8$ à $7,7 \mathrm{p}$. Ioo. Ce dernier résultat est vraisemblablement lié au développement moins important des brebis nées quadruples.

\section{TABLEAU 4}

Infuence de l'âge des brebis et de leur mode de naissance sur la prolificité (SMIRNOV, I935)

\begin{tabular}{|c|c|c|c|c|c|c|c|c|c|c|c|c|}
\hline \multirow{2}{*}{$\begin{array}{l}\text { Mode de naissance } \\
\text { Simples .......... }\end{array}$} & \multicolumn{2}{|c|}{$1^{\text {re }}$ mise bas } & \multicolumn{2}{|c|}{$2^{\mathrm{e}}$ mise bas } & \multicolumn{2}{|c|}{$3^{\mathrm{e}}$ mise bas } & \multicolumn{2}{|c|}{$4_{4} \mathrm{e}$ mise bas } & \multicolumn{2}{|c|}{$5^{\mathrm{e}}$ mise bas } & \multicolumn{2}{|c|}{$6^{\mathbf{e}}$ mise bas } \\
\hline & $(32)$ & 1,78 & $(45)$ & 1,97 & (39) & 2,07 & $(27)$ & 2,48 & (26) & 2,46 & (5) & 1,80 \\
\hline Doubles $\ldots \ldots \ldots$ & $(112)$ & 1,83 & $(160)$ & 2,06 & $(1 / 4)$ & 2,15 & $(88)$ & 2,34 & $(100)$ & 2,46 & $(32)$ & 2,41 \\
\hline 'Triples $\ldots \ldots$ & $(35)$ & 1,79 & $(93)$ & 2,00 & $(85)$ & $2, \geq 6$ & $(55)$ & 2,34 & $(41)$ & 2,50 & $(25)$ & 3,26 \\
\hline Quadruples & $\begin{array}{l}(22) \\
\vdots\end{array}$ & $1,8^{\prime}$ & (29) & 2,37 & $(22)$ & 2,31 & $(15)$ & 2,40 & (14) & 2,68 & $(10)$ & 3,00 \\
\hline
\end{tabular}

Le chiffre entre parenthèses indique le nombre de brebis observées.

Par ailleurs, les auteurs mettent en évidence de nombreux facteurs influant sur ce caractère, parmi lesquels l'âge des brebis, l'époque d'agnelage et l'état des brebis au moment de la lutte :

- Dobrotvorskaia (I965) indique des taux de I,79 et r,90 pour des agnelles mises à la lutte respectivement à IO-I I mois et à I 8 mois. SMIRnov (I935) montre (tabl. 4) que la prolificité augmente jusque vers le $5^{\text {e }}$ agnelage. Les observations de Kovnerev et al. (1967) sont similaires pui sque 62 brebis donnant 2, I 3 agneaux en première mise bas ont donné aux $4^{e}, 5^{\mathrm{e}}$ et $6^{\mathrm{e}}$ agnclage 2,63 agneaux et aux 9 et roe, 2,26 agneaux. 
- Comme pour les autres races, l'agnelage de printemps donne de meilleurs résultats que l'agnelage d'automne; KovNerev et al. (I967) observent en agnelage de février-mars, une prolificité de 2,72 contre 2,05 en septembre-octobre (respectivement sur $24 \mathrm{I}$ et 2 I 2 brebis). De même

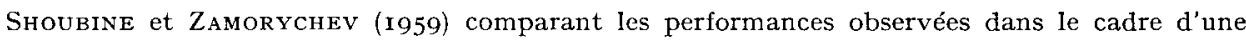
expérience de double agnelage donnent les résultats suivants :

- agnelage d'automne et de printemps : 2,24 et 2,26;

- agnelage d'hiver et d'été : 2,09 et 1,84 ;

- le taux d'agnelage des brebis mal développées est inférieur à celui des brebis de plus grand format. Ainsi, Smirnov (1935) note que dans un même élevage, les brebis pesant de 55 à $65 \mathrm{~kg}$ donnent une moyenne de 2,5 agneaux contre $\mathrm{I}, 62$ pour celles pesant entre $25 \mathrm{et} 30 \mathrm{~kg}$. De même, Kovnerev ( 1963 ) indique que les brebis en bon état donnent 8 à I 6 p. Ioo d'agneaux de plus que les brebis en mauvais état. De son côté, Pokrovskaia (I957) rapporte que parmi des brebis supplémentées avant la lutte, $56 \mathrm{p}$. Ioo donnent des portées triples et 33 p. roo des portées doubles alors que des brebis non complémentées donnent toutes des doubles.

\section{4. - Durée de l'estrus}

Plusieurs auteurs signalent la liaison existant entre la durée de l'œstrus et le taux de prolificité correspondant :

- Polikarpova, Zelenski et Nevzgodina (1963) observent des chaleurs qui durent en moyenne $7^{2}$ heures consécutives, $2,7 \mathrm{p}$. Ioo des brebis présentant un ostrus de 5 à 6 jours. Ils montrent que ce sont les brebis dont l'œstrus dure trois jours et plus qui ont les taux de prolificité les plus élevés (Polikarpova, I964).

- Guerassimov (I957) avait observé un phénomène analogue qu'il liait au format des brebis : le tableau 5 indique pour 300 brebis ayant une durée moyenne d'œstrus de 58,6 heures et un taux de prolificité de 2 I9,o, les valeurs de ces deux critères en fonction du poids vif au moment de la lutte.

\section{TABLEAU 5}

Influence du poids vif des brebis sur la durée de l'astrus et sur la prolificité (Guerassimov, I957)

\begin{tabular}{l|r|r|c}
\hline \hline Poids vif $(\mathrm{kg})$ & Nombre de brebis & Durée d'œstrus (h) & Prolificité \\
& & & \\
\hline & & & \\
25 à 30 & 49 & 45,6 & \\
31 à 36 & 107 & 53,6 & 1,666 \\
37 à 42 & 89 & 59,0 & 2,042 \\
43 à 48 & $3 \prime$ & 64,7 & 2,113 \\
49 à 54 & 12 & 61,2 & 2,360 \\
55 à 60 & & 67,2 & 2,236 \\
& & & 2,500 \\
\hline
\end{tabular}

Les auteurs ci-dessus considèrent que la durée de l'œstrus est proportionnelle au nombre de follicules et à leur vitesse de maturation ; ils estiment que l'ovulation est espacée dans le temps et que les chaleurs cessent 6 à 8 heures après la dernière ponte ovulaire.

La technique de lutte libre nécessiterait de ce fait l'utilisation d'un grand nombre de béliers pour éviter leur épuisement qui entraînerait un étalement de l'agnelage et une baisse des taux de fertilité et de prolificité. Guerassimov (I957) note que la prolificité augmente de I,98 à 2,40 quand le nombre de saillies passe de i à 5. De même, Polikarpova, Zelensky et Nevzgodina (1963) montrent que les brebis saillies 2 fois et plus ont une prolificité de 2,75 contre 2, Io pour celles saillies une seule fois. Ils recommandent en conséquence le recours à la lutte en main ou 
à l'insémination artificielle quotidienne (ou biquotidienne) pendant toute la durée de l'œstrus pour assurer la fécondation des ovules au fur et à mesure de leur libération. Un troupeau pourrait ainsi être inséminé au cours d'une période de $I_{5}$ à 20 jours, et réaliser l'agnelage dans un laps de temps presque aussi restreint.

Les différentes méthodes de lutte permettent peut-être d'expliquer des taux de fertilité très variables selon les auteurs : alors que Peregone (1950) considère que seulement $83 \mathrm{p}$. Ioo des brebis sont gestantes après la lutte, Kovnerev (1955) annonce 90,4 p. Ioo de réussite sur I 25 brebis et GoRB (1945) 99 p. Ioo sur 588 brebis.

\section{5. - Rusticité des mères et longévité}

Seltanine (I957) indique une mortalité de i6,9 p. I00 che $z$ les brebis adultes, dont les causes seraient essentiellement les maladies pulmonaires et les parasites intestinaux. Ces taux sont très différents suivant le mode d'élevage puisque Youdine et Petrov (I957) signalent une mortalité de Io à I I p. Ioo pour des brebis élevées en bergerie contre 3,7 à $7,6 \mathrm{p}$. roo pour des brebis conduites en plein air.

Kovnerev (1965) estime que l'âge limite de réforme se situe vers 7 I/2-8 ans, et SEliaNINE (1965) chiffre la longévité des brebis au cours de l'expérience de comparaison de mise à la lutte d'agnelles de différents âges, citée plus haut (tabl. 6). Il semble qu'une mise en lutte précoce des agnelles soit préjudiciable à leur longévité.

\section{TABI,EAU 6}

Influence de l'âge à la première lutte sur la longévité des brebis

(Selianine, I965)

\begin{tabular}{|c|c|c|c|c|}
\hline & \multirow{2}{*}{$\begin{array}{l}\text { Lutte } \\
\text { à } 18 \text { mois }\end{array}$} & \multirow{2}{*}{$\begin{array}{l}\text { Lutte } \\
\text { à } 6-7 \text { mois }\end{array}$} & \multicolumn{2}{|c|}{ Lutte à 10-11 mois } \\
\hline & & & $\begin{array}{l}\text { Alimentation } \\
\text { poussée }\end{array}$ & $\begin{array}{l}\text { Alimentation } \\
\text { normale }\end{array}$ \\
\hline Nombre de brebis $\ldots \ldots \ldots \ldots \ldots$ & 280 & $6 / 4$ & 26 & 31 \\
\hline Nbre d'années de production $(\%)$ : & & & & \\
\hline 2 ans $\ldots \ldots \ldots \ldots \ldots \ldots \ldots \ldots$ & 6,1 & 13,4 & 4,1 & 4,8 \\
\hline 3 à 5 ans $\ldots \ldots \ldots \ldots \ldots$ & 28,3 & 36,1 & 25,2 & 28,9 \\
\hline 6 à 7 ans $\ldots \ldots \ldots \ldots \ldots$ & 49,4 & 44,2 & 52,7 & 47,0 \\
\hline 8 ans et plus $\ldots \ldots \ldots$ & 16,3 & 6,3 & 17,0 & 12,3 \\
\hline Nombre moyen d'agnelages .. & 7,6 & 7,5 & 8,0 & 7,3 \\
\hline
\end{tabular}

\section{III. - CARACTÈRES D'ÉLEVAGE DES AGNEAUX E'T VAI,EUR BOUCHÈRE}

\section{I. - Mortalité des agneaux}

Nous observons de façon générale un taux de mortalité plus élevé chez les agneaux multiples que chez les agneaux simples; il paraît donc intéressant de voir quelles sont les estimations données par les auteurs pour cette race :

Panine (I957) et Selianine (1957) enregistrent des mortalités pouvant atteindre dans les extrêmes des taux de $4^{\circ}$ à $5^{\circ} \mathrm{p}$. Ioo essentiellement dues aux maladies pulmonaires et aux para- 
sites intestinaux, et liées à une alimentation insuffisante. Mais en moyenne, pour un taux de prolificité de $\mathrm{I}, 78$ à $\mathrm{I}, 83, \mathrm{I}, 37$ à $\mathrm{I}, 38$ agneaux sont sevrés pour Ioo mères.

En fait le mode d'élevage et l'époque d'agnelage sont susceptibles d'influer sur la vigueur des agneaux :

- Youdine et Petrov (I957) signalent des taux de mortalité de $4^{8}$ p. ioo en bergerie contre 3,7 à 6,4 p. Ioo en élevage de plein air. D'autre part, SMIrnov et Sveco (I94I) comparant deux lots de I oo brebis conduites, les unes en troupeaux, les autres en cases individuelles, constatent des pertes jusqu'au sevrage qui sont respectivement de 6,70 p. Ioo et de I3,67 p. I00 pour des taux de prolificité sensiblement voisins, de l'ordre de 2,0 .

Smirnov, Smirnova et Voskovoinikov (I954) et Guerassimov (I957) mettent en évidence une mortalité moindre pour les agneaux nés à l'automne que pour ceux nés en hiver. SELIANINE (1957) cite des pertes de 4,7 p. roo en agnelage d'hiver. Ces auteurs attribuent ces différences au fait que les mères sont mal nourries à la fin de l'automne, période qui correspond à la fin de la gestation dans le cas d'un agnelage d'hiver : cela entraîne un poids des agneaux à la naissance plus faible, une vigueur moindre et une production laitière des brebis insuffisante. KovNEREV et al. (1967) mettent également ce phénomène en évidence : tableau 7.

\section{TABLEAU 7}

Influences de l'époque de lutte sur le poids vif et la prolificité des brebis, et sur le poids à la naissance, la croissance et la vitalité des agneaux

(Kovnerev et al., I967)

\begin{tabular}{|c|c|c|}
\hline & Lutte avril-mai & Lutte août-septembre \\
\hline 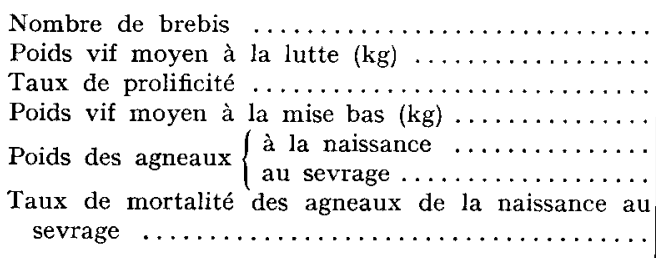 & $\begin{array}{l}212 \\
41,6 \\
2,05 \\
46,8 \\
2,76 \\
18,4 \\
1,7\end{array}$ & $\begin{array}{l}241 \\
47,3 \\
2,72 \\
42,4 \\
2,24 \\
15,3 \\
12,2\end{array}$ \\
\hline
\end{tabular}

\section{2. - Poids à la naissance}

Le tableau 8 regroupe les estimations du poids à la naissance réalisées par différents auteurs selon le sexe des agneaux et la taille de la portée dont ils sont issus.

Les variations observées sont en outre imputables à différents effets :

- les agneaux nés de brebis jeunes ou très âgées sont plus légers que ceux nés de brebis d'âge moyen : Selianine (1958);

- l'époque d'agnelage influe sur le poids à la naissance des agneaux du fait des conditions d'alimentation des brebis en fin de gestation : Smirnov, Smirnova et Voskovolnikov (I954) et Guerassimov (1957) indiquent que les agneaux nés à l'automne sont plus lourds que ceux nés en hiver. Si l'agnelage est encore plus tardif, la différence s'accroît : Pokrovskaia (I957) signale que des agneaux nés en février-mars pèsent en moyenne $0,5 \mathrm{à} 0,7 \mathrm{~kg}$ de moins que des agneaux nés en décembre-janvier.

Ce dernier auteur met en évidence l'influence du niveau d'alimentation des brebis puisque le poids à la naissance des agneaux est respectivement de 3,2,3,2,4 $\mathrm{kg}$ pour les simples, doubles et triples nés de brebis complémentées en fin de gestation contre 2, $1,65,1,52 \mathrm{~kg}$ pour les agneaux de ces 3 catégories issus de mères non complémentées. 
TABLEAU 8

Poids à la naissance des agneaux selon leur sexe et leur mode de naissance

\begin{tabular}{|c|c|c|c|c|}
\hline Type d'agneaux & Lffectif & $\begin{array}{l}\text { Moyenne } \\
\quad(\mathrm{kg})\end{array}$ & $\begin{array}{l}\text { Valeurs } \\
\text { extrêmes }\end{array}$ & Auteur \\
\hline Mâles & 110 & 2,36 & & Kovnerev \\
\hline Femelles & 105 & $2,2 / 1$ & & Kovnerev \\
\hline Simples & -7 & $\begin{array}{l}3,16 \\
2,8\end{array}$ & $2,1 \grave{a} 3,8$ & $\begin{array}{l}\text { Gorb } \\
\text { Mekhtiev }\end{array}$ \\
\hline Doubles & $\frac{36}{-}$ & $\begin{array}{l}1,9 \\
2,6^{\prime} \mathbf{t} \\
2,3\end{array}$ & $\begin{array}{l}1,1 \text { à } 2,1 \\
\frac{-}{1,5} \text { à } 2,9\end{array}$ & $\begin{array}{l}\text { StarovoITENKo } \\
\text { GorB } \\
\text { MEKHTIEV }\end{array}$ \\
\hline Triples & $\begin{array}{l}38 \\
11\end{array}$ & $\begin{array}{l}1,1 \\
1,7\end{array}$ & $\begin{array}{l}1,3 \text { à } 2,0 \\
1,3 \text { à } 2,4\end{array}$ & $\begin{array}{l}\text { STaROVOTTENKO } \\
\text { MEKHTIEV }\end{array}$ \\
\hline Quadruples & 16 & 0,9 & 0,7 à 1,5 & StaRovoITENKO \\
\hline
\end{tabular}

\section{3. - Production laitière des brebis}

La croissance des agneaux sera dans un premier temps fonction de la production laitière des brebis. Signalée comme élevée par Louzina (1943) et Panine (I957) elle est chiffrée par Smirnova (1958) entre I I et $157 \mathrm{~kg}$ de lait pour une lactation de roo jours avec une teneur en matière grasse variant de 6,27 à 7,35 p. Ioo (tabl. 9). Cet auteur indique que la production

\section{TABLEAU 9}

Production laitière et composition du lait à différents stades de la lactation (d'après Smirnova, I958).

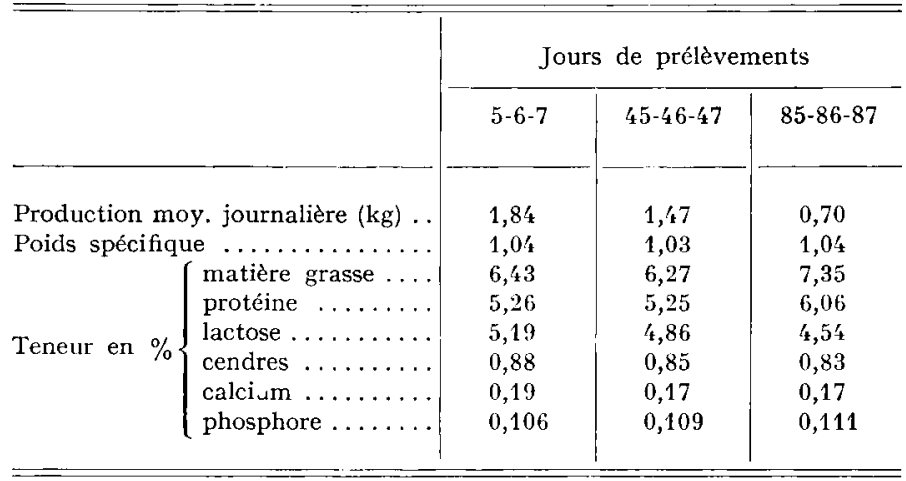

laitière augmente rapidement pour atteindre son maximum à 15 jours, et baisse ensuite progressivement jusqu'au sevrage. Elle serait plus élevée chez la brebis ayant 6 à 7 ans. KovNEREv (1966) note par ailleurs l'existence de lignécs possédant 4 mamelles fonctionnelles. 
KovNEREv et al. (1967) estiment qu'en 20 jours d'allaitement, $5^{\circ}$ p. Ioo des brebis assurent à leur portée un gain de poids de $\mathrm{I}_{2}$ à $\mathrm{I} 4 \mathrm{~kg}$, ce qui représenterait 52 à $60 \mathrm{~kg}$ de lait, soit 2,6 à $3 \mathrm{~kg}$ de lait par jour. Ces estimations sont basées sur un coefficient de transformation de $4,3 \mathrm{~kg}$ de lait pour I kg de croît alors que SEMENTSCHENko (1952) propose des coefficients de transformation de 3,84 pour les mâles et 4,I 8 pour les femelles. Des observations plus précises réalisées par ZaMorycheVA (KovNerev et al., 1967) montrent l'influence du mode et du niveau d'alimentation sur la production laitière: (tabl. Io).

\section{TABLEAU IO}

Infuence du mode d'alimentation des brebis sur leur production laitière (d'après ZAMORYcheva et KovNEREv et al., I967)

\begin{tabular}{|c|c|c|c|c|c|c|c|c|}
\hline \multirow{2}{*}{$\frac{\text { Époque }}{\text { Product. laitière }(\mathrm{kg})}$} & \multicolumn{2}{|c|}{$1^{\text {er }}$ mois } & \multicolumn{2}{|c|}{$2^{\mathrm{e}}$ mois } & \multicolumn{2}{|c|}{$3^{\mathrm{e}}$ mois } & \multicolumn{2}{|c|}{ En 90 jours } \\
\hline & totale & journalière & totale & journalière & totale & journalière & totale & journalière \\
\hline $\begin{array}{r}\text { Alimentat. améliorée : } \\
\text { au pâturage....... }\end{array}$ & 58,9 & 1,96 & 44,2 & 1,47 & 27,6 & 0,90 & 130,7 & 1,45 \\
\hline en bergerie..... & 56,9 & 1,89 & 38,1 & 1,27 & 29,6 & 0,98 & 124,6 & 1,38 \\
\hline $\begin{array}{l}\text { Alimentat. modérée : } \\
\text { au pâturage ...... }\end{array}$ & 43,6 & 1.44 & 36.7 & 1,22 & 204 & 0.78 & 1 & 115 \\
\hline en bergerie ........ & 45,3 & 1,51 & 34,8 & 1,14 & 20,4 & 0,67 & 99,8 & 1,10 \\
\hline
\end{tabular}

Enfin, Sementschenko (1952) met en évidence la persistance de la lactation chez la brebis allaitant plusieurs agneaux, les mères d'agneaux triples produisant encore $\mathrm{r}, 03 \mathrm{~kg}$ de lait à roo jours contre $0,69 \mathrm{~kg}$ pour les mères d'agneaux doubles.

\section{4. - Croissance des agneaux}

Compte tenu du fait que les brebis doivent allaiter 2 à 3 agneaux en moyenne, la croissance des agneaux ne peut atteindre les valeurs observées chez nos races moins prolifiques. Sur ıoo mâles et Ioo femelles, Kovnerev (I955) observe des moyennes respectives de $5,83 \mathrm{~kg}$ et $5,80 \mathrm{~kg}$ à 20 jours et $\mathrm{I} 7,03$ et $\mathrm{I} 6,73 \mathrm{~kg}$ à trois mois; de même Mojaeva (1965) et Sementschenko (1952) avancent des poids moyens de 18 à $20 \mathrm{~kg}$ au sevrage à quatre mois.

Aussi Starovortenko et Eline (1965) préconisent-ils une supplémentation précoce des agneaux sous forme de lait de vache à raison de 150 à $250 \mathrm{~g}$ par jour dès la deuxième semaine, et de grain écrasé (I oo à $200 \mathrm{~g}$ par jour) à partir de 45 jours, soit un apport total jusqu'au sevrage de $\mathbf{2} 2 \mathrm{~kg}$ de lait et $\mathrm{I} 2 \mathrm{~kg}$ de grain par agneau. C'est sans doute avec une telle supplémentation que Pokrovskaia (I957) observe des gains moyens journaliers atteignant $300 \mathrm{~g}$.

Après le sevrage, la croissance des agneaux dépend essentiellement des conditions d'alimentation. Si Selianine et Koulikov (I954) indiquent des poids de $22-27 \mathrm{~kg}$ à $5-6$ mois et $27-34 \mathrm{~kg}$ à 8-Io mois, d'autres auteurs annoncent que des agnelles convenablement nourries atteignent $40 \mathrm{~kg}$ vers 6 à 8 mois (PANine, 1957), 40 à $45 \mathrm{~kg}$ à 9-Io mois (KovNerev, I965). Ainsi, ZamoryCHEV (I963) constate, au cours d'une période d'engraissement de izo jours après le sevrage, des gains moyens journaliers de ${ }^{7} 73 \mathrm{~g}$ sur pâture naturelle. Starovortenko et Eline (I965), nourrissant les agneaux avec de l'ensilage et du foin ad libitum et $300 \mathrm{~g}$ de grain écrasé pendant trois mois, puis sur pâturage avec complément de pois et d'avoine, obtiennent des croissances de $240,210,205 \mathrm{~g} / \mathrm{j}$ respectivement pour les doubles, triples et quadruples; les poids moyens 
à 8 mois atteignent $37, \mathrm{I}, 36,5$ et $36,0 \mathrm{~kg}$ avec des valeurs extrêmes de 47,45 et $43 \mathrm{~kg}$. D'après Kovnerev et al. (I967) l'effet du poids à la naissance sur la croissance des agnelles resterait sensible jusque vers 3 ans, comme le montre le tableau i I.

TABLEAU II

Poids vif des agnelles à différents âges selon leur poids à la naissance. (KovNEREV et al., I967)

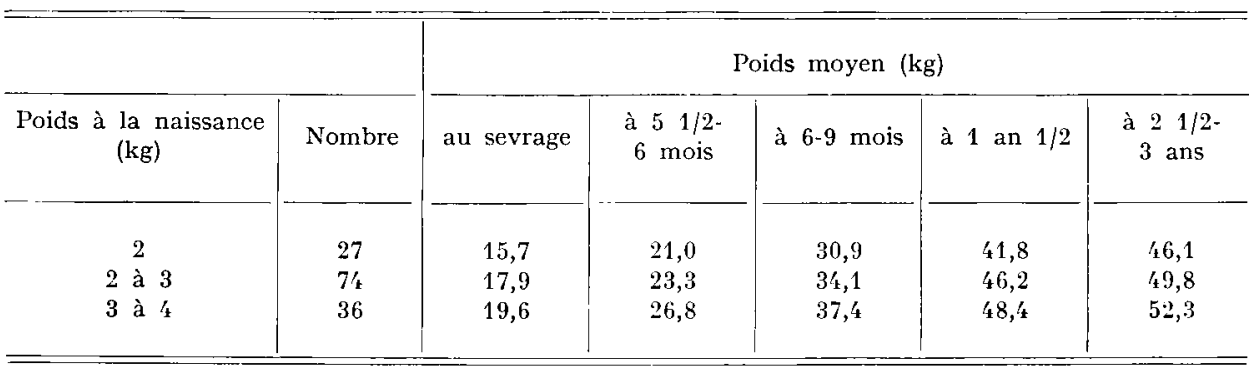

\section{5. - Poids des adultes}

Les estimations du poids des animaux adultes sont assez variables (tabl. I2). Ces différences sont vraisemblablement liées au fait que la croissance se poursuit jusqu'à l'âge de 5 à 7 ans. PANine (1957) obtient, quant à lui, une moyenne de $45,4 \mathrm{~kg}$ sur un échantillon de 3550 brebis, et il montre l'intérêt d'une sélection sur le format des animaux en mettant en évidence une corrélation positive de 0,28 entre le poids vif des brebis et leur prolificité.

TABIEAU I 2

Poids vif des adultes (kg)

\begin{tabular}{|c|c|c|c|}
\hline Auteur & Brebis & Béliers & Observations \\
\hline SZYMSKI, 1954 & 42 & 50 & à 20 mois \\
\hline HEPTNER, 1941 & 48 à 52 & 57 & \\
\hline Selianine, 1960 & 42 & & \\
\hline DOBROTVORSKAIA, 1965 & 46 à 47 & & à 2 ans \\
\hline MoJAEVA, 1965 & 45 & 55 & $\begin{array}{c}\text { élevages } \\
\text { traditionnels }\end{array}$ \\
\hline Kovnerev, 1966 & 50 à 55 & 80 à 100 & fermes pilotes \\
\hline \multirow{2}{*}{ KoVnerev, 1965} & 42 & & $\begin{array}{l}\text { brebis de réforme } \\
\text { avant engraissement }\end{array}$ \\
\hline & 54,5 & & $\begin{array}{l}\text { brebis de réforme } \\
\text { après engraissement }\end{array}$ \\
\hline Sementschenko & 50,8 & 70 & \\
\hline
\end{tabular}


Par ailleurs, il est certain que l'époque de la pesée a une grande importance, puisque SEMENTSCHENKo (I952) observe jusqu'à $7 \mathrm{~kg}$ de perte de poids au cours de la lactation pour des brebis ayant une production laitière élevée, nourrissant des triplés.

\section{6. - Age à l'abattage des agneaux et valeur bouchère}

Alors que traditionnellement les agneaux étaient abattus au-delà d'un an, différents auteurs préconisent un abattage plus précoce se situant entre 5 et 8 mois : Zelensky et Troussova (1958); Zamorychev !(1963); IAdRilev et Stepanova (1965). Les raisons invoquées par Youdine et Petrov (1957), Selianine et Koulikov ( 1964 ) en sont l'obtention d'une viande plus jeune d'une part, et d'une fourrure ayant une valeur bien supérieure d'autre part.

Le rendement à l'abattage varie suivant l'âge et les conditions d'alimentation. Pour des agneaux de 7 à 8 mois, ZAMORYCHEV (1963) indique des rendements de l'ordre de 49 p. IOo alors que IAdRILEv et Stepanova (1965) obtiennent des rendements de 42 p. Ioo au même âge. KovneREV (I965) observe pour 9 mâles caștrés soumis à un régime élevé et abattus à I 3 mois et demi, des rendements de $57,7 \mathrm{p}$. Ioo pour un poids vif à jeun de $53,7 \mathrm{~kg}$ : ces carcasses comportaient $5,5^{2} \mathrm{~kg}$ de gras interne et avaient la composition suivante : 19,6 p. Ioo de gras; i6, I p. Ioo de protéines, o,69 p. Ioo de cendres et $63,6 \mathrm{p}$. Ioo d'eau.

Les morceaux nobles représentent 60 à $70 \mathrm{p}$. 100 du poids de carcasse contre 20 à $25 \mathrm{p}$. Ioo pour les morceaux de $2^{\mathrm{e}}$ qualité (cou, poitrine, aine) et $\mathrm{s}$ à 15 p. Ioo pour les morceaux de $3^{\text {e }}$ qualité.

Du fait de l'ossature peu développée des animaux de cette race, le pourcentage de viande nette dans la carcasse atteint 80 p. Ioo. Selianine et Koulikov ( 1964$)$ ont comparé les qualités de carcasses de 350 agneaux répartis en trois groupes, le premier abattu à 5-6 mois, le deuxième à 8-9 mois après une alimentation normale, le troisième à $8-9$ mois à la suite d'un haut régime alimentaire (tabl. I3) ; les rendements obtenus sont respectivement de 39,8, 42,6 et 44,9 p. Ioo.

TABLEAU I3

Influence de l'âge d'abattage et du niveau d'alimentation des agneaux sur la composition corporelle (Selianine et Koulikov, 1964)

\begin{tabular}{|c|c|c|c|c|c|c|}
\hline & \multicolumn{2}{|c|}{ Abattage à $5-6$ mois } & \multicolumn{4}{|c|}{ Abattage à $8-9$ mois } \\
\hline & \multirow[b]{2}{*}{ Moyenne } & \multirow{2}{*}{$\begin{array}{c}\text { Limites } \\
\text { de variations }\end{array}$} & \multicolumn{2}{|c|}{ Alimentation normale } & \multicolumn{2}{|c|}{ Alimentation poussée } \\
\hline & & & Moyenne & $\mid \begin{array}{c}\text { Limites } \\
\text { de } \\
\text { variations }\end{array}$ & Moyenne & $\begin{array}{c}\text { Limites } \\
\text { de variations }\end{array}$ \\
\hline Poids vif à l'abattage & 21,8 & $15,5-26,5$ & 25,6 & $22,0-31,0$ & 29,6 & $25,0-38,0$ \\
\hline Poids de $\left\{\begin{array}{l}\text { carcasse } \ldots \ldots \ldots \ldots \\
\text { muscles } \ldots \ldots \ldots \ldots \\
\text { gras } \ldots \ldots \ldots \ldots \ldots \\
\text { os et tendon } \ldots \ldots\end{array}\right.$ & $\begin{array}{l}8,67 \\
5,85 \\
0,40 \\
2,12\end{array}$ & $\begin{array}{l}5,3-10,1 \\
3,7-7,7 \\
0,1-0,6 \\
1,8-2,9\end{array}$ & $\begin{array}{l}10,9 \\
8,20 \\
0,24 \\
2,50\end{array}$ & $\begin{array}{r}8,0-15,8 \\
5,7-12,2 \\
0,15-0,33 \\
1,9-3,1\end{array}$ & $\begin{array}{l}13,3 \\
9,80 \\
0,68 \\
2,82\end{array}$ & $\begin{array}{c}10,0-18,0 \\
7,4-14,1 \\
0,4-0,97 \\
2,5-3,0\end{array}$ \\
\hline 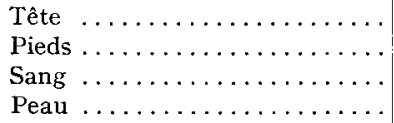 & $\begin{array}{l}1,15 \\
0,56 \\
0,65 \\
1,85\end{array}$ & $\begin{array}{l}0,8-1,3 \\
0,4-0,9 \\
0,5-0,9 \\
1,3-2,5\end{array}$ & $\begin{array}{l}1,16 \\
0,64 \\
0,66 \\
2,49\end{array}$ & $\begin{array}{l}0,7-2,1 \\
0,4-1,2 \\
0,6-0,8 \\
2,1-3,6\end{array}$ & $\begin{array}{l}1,30 \\
0,80 \\
0,80 \\
2,76\end{array}$ & $\begin{array}{l}1,0-2,1 \\
0,6-1,2 \\
0,7-0,9 \\
2,1-3,7\end{array}$ \\
\hline
\end{tabular}


Notons que Melerine et Arzoumaniane (1965) proposent des équations de régression permettant d'estimer le poids du squelette ( $\mathrm{X}$ en grammes) à partir du volume du canon $(Z)$ mesuré par son diamètre et sa longueur :

$$
X=336,6+77,2 Z-\mathbf{1}, 2 Z^{2}
$$

et le poids de muscle ( $\mathrm{Y}$ en $\mathrm{g}$ ) à partir du poids vif ( $\mathrm{W}$ en $\mathrm{kg}$ ) :

$$
\mathrm{Y}=228 \mathrm{~W}+4^{86}
$$

Les erreurs sont respectivement de 0,3 à 5,4 p. Ioo et de 0,7 à 8, I p. Ioo dans le cas de leur échantillon qui se limitait malheureusement à i I agneaux.

En fin, KovNEREV et al. $(1967)$ affirment que contrairement aux autres races ovines exploitées en U. R. S. S., le Romanov n'a pas le goût de suint.

\title{
IV. - MÉTHODES D'ÉLEVAGE PRÉCONISÉES
}

\author{
I. - Conduite du troupeau
}

Les auteurs s'accordent à montrer la supériorité de l'élevage en plein air sur l'élevage en bergerie tant au point de vue de l'état sanitaire que des caractères de production : poids vif, prolificité, toison (Kovnerev, I958). En particulier, Starkova (cité par Panine, I957) constate une vitesse de croissance supérieure chez des agneaux élevés en plein air dès la naissance par des températures atteignant $-{ }_{1} 5^{\circ} \mathrm{C}$ par rapport à des agneaux élevés dans des bergeries à $+2^{\circ} \mathrm{C}$ at des taux de mortalité respectivement de 5,7 et $20 \mathrm{p}$. roo. Kharitonov (cité par Panine, 1957) signale une meilleure minéralisation des os chez des animaux de plein air. En fin, SELIANINE (I957) conseille d'éviter les bergeries chaudes et humides; il préconise l'emploi d'enclos bardés du côté des vents dominants en période d'agnelage et pendant les grands froids.

\section{2. - Castration des agneaux mâles}

Alors que Panine (I957) est partisan d'une castration des agneaux à 2 ou 3 semaines, KovneRev et Mosiaguina (I963), Devine, Kandalov et Nasonovsky (I963) comparant la croissance et la valeur bouchère d'agneaux entiers, castrés ou vasectomisés, constatent des performances similaires jusqu'à l'âge de 6 mois; les seules différences apparaissent au niveau de la toison, les agneaux entiers ou vasectomisés présentant une crinière jarreuse importante qui est un caractère sexuel secondaire. En définitive, la castration n'est pas recommandée si les agneaux sont abattus à un âge relativement précoce.

\section{3. - Alimentation}

C'est sur ce plan que les auteurs préconisent le plus de soins; nous avons montré précédemment l'influence du niveau alimentaire sur les performances des brebis et sur la croissance des agneaux.

Citons en particulier l'expérience de Zelensky et Troussova (I958) qui comparent deux lots d'agneaux élevés de 3 à 5 mois sur pâturage d'une part sans complément, d'autre part avec $2 \mathrm{~kg}$ de mélange de vesce-avoine, par tête et par jour (tabl. I4) : le gain moyen journalier des agneaux passe de 163 à 220 grammes, et le rendement en carcasse de 42,5 à 52,2 p. roo.

Plus récemment, IAdrilev et Stepanova (I965) engraissant deux lots de mâles en pâture, l'un avec $200 \mathrm{~g}$ de concentré par tête et par jour, l'autre avec $300 \mathrm{~g}$ obtiennent les résultats portés au tableau I5 $_{5}$ Le premier lot, abattu à I $3^{-1} 4$ mois a entraîné une perte économique alors que le second, abattu à 7-8 mois a assuré un bénéfice.

Pour des brebis en fin de gestation, Pokrovskaia (1957) préconise une ration assurant en moyenne un apport énergétique de $\mathrm{I}, 6$ unités fourragères et un apport azoté de $150 \mathrm{~g}$ de matière digestible (tabl. I6). Pour des brebis allaitantes, ce même auteur conseille d'ajouter $200 \mathrm{~g}$ de concentré et 4 oo g d'aliments succulents par agneau élevé. 


\section{TABLEAU I4}

Comparaison de la croissance, du poids de carcasse et du rendement d'agneaux au pâturage avec ou sans complémentation (ZELENSKY et Troussova, I958)

\begin{tabular}{l|c|c}
\hline \hline & Lot témoin & Lot complémenté \\
\hline Poids en début d'expérience .... & 16,3 & 16,5 \\
\hline Poids avant l'abattage ....... & 26,1 & 29,7 \\
\hline Poids de carcasse........... & 11,1 & 15,5 \\
\hline Rendement ............... & 42,5 & 52,2 \\
\hline \hline
\end{tabular}

\section{TABLEAU I5}

Comparaison de la vitesse de croissance et de l'indice de consommation selon deux niveaux de complémentation

(IADRILEV et STEPANOVA, I965)

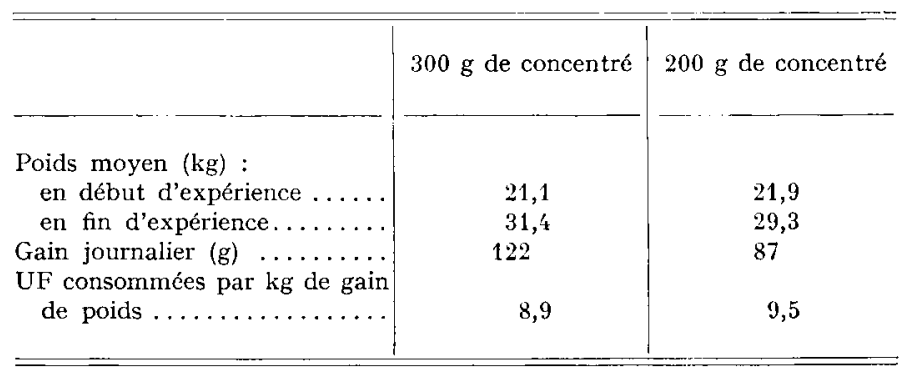

TABLEAU I6

Ration journalière pour des brebis en fin de gestation (Pokrovskaia, I957)

\begin{tabular}{l|c|c|c}
\hline & Quantité $(\mathrm{kg})$ & $\begin{array}{c}\text { Unités } \\
\text { fourragères }\end{array}$ & M.A.D. (g) \\
\hline $\begin{array}{l}\text { Foin de trèfle et d'herbe... } \\
\text { Concentré (avoine, son, } \\
\begin{array}{c}\text { Ensilage, betteraves, pom- } \\
\text { mes de terre ........... }\end{array}\end{array}$ & 1,8 & 0,94 & 58 à 99 \\
\hline
\end{tabular}




\title{
V. - LE " ROMANOV " E'T LES CROISEMENTS
}

\author{
I. - Amétioration du Romanov par croisements
}

Dès I 845, Mikelsone considère à l'issue d'essais d'infusion de sang Southdown ou Mérinos que le croisement, s'il améliore les qualités bouchères ou lainières, détériore les qualités de prolificité et de fourrure de la race. D'autres essais réalisés avec le Mérinos (Gavrilov, r88o), avec le Southdown et le Border Leicester en I9or, avec le Lincoln en Igro et I923-1924 (KovNeREv et al., 1967) et avec le Shropshive (Louzina, 1943) n'ont pas donné de résultats plus satisfaisants.

\section{2. - Amélioration de différentes races par le Romanov}

En I934, Bounkine et al., signalent l'intérêt qu'il y aurait à utiliser le Romanov pour améliorer la qualité de fourrure des agneaux dans différentes races. Au cours de la même année, et plus récemment en 1953, la Pologne a importé des Romanov utilisés en croisement avec la race locale Wrzoswka (race des bruyères). Szymski (1954) rapporte que cette infusion de sang a permis d'améliorer à la fois la qualité des fourrures des agneaux et la prolificité de la race locale.

En I933, Nikolsky envisage l'utilisation de béliers Romanov pour améliorer la prolificité de la race Karakul; l'expérience est réalisée à Askania Nova à partir de 1935 et relatée par Peregone (I950); 508 Karakul et I4I Romanov sont soumis à des croisements réciproques, les produits étant croisés en retour avec des Karakul. Les individus issus de ce " back cross " ont ensuite été accouplés entre eux au cours des générations successives et sélectionnés sur la prolificité. A partir de données portant sur 9692 agneaux au cours de 6 générations successives Peregone montre que cette nouvelle souche, dénommée "Karakul multifœtal ", a une prolificité de 42 à 54 p. Ioo supérieure à celle des Karakul purs. A Askania Nova, 50 à 70 p. too des brebis donnent des doubles et 3 à 5 p. I oo donnent des triples (soit une prolificité de I, 56 à I, 80). Parallèlement, le taux de brebis vides est réduit de I 7 p. Ioo et 8,8 p. Ioo respectivement chez le Romanov et le Karakul, à 7,4 p. Ioo dans cette souche. De plus, le poids à la naissance des agneaux reste élevé puisque il varie de $4 \mathrm{~kg}$ à $4,4 \mathrm{~kg}$ chez le Karakul multifotal contre 4 à 4,6 en Karakul, et 2 à 2,4 en Romanov. En I952, le Karakul multifotal est considéré comme une nouvelle race (PEREGONE, 1953) présentant une prolificité moyenne de 1,64 et des caractères de fourrure tout à fait semblable à ceux des Karakul.

Golovina (1936) rapporte les résultats d'une expérience de croisement entre des femelles de la race nordique à queue courte et des béliers Romanov; cet auteur conclut à une amélioration du format des animaux et des qualités de la fourrure par l'apport du sang Romanov. Une expérience analogue de croisement avec la race nordique à queue courte dans la république des Komis en 1949 et r950 permet d'obtenir des brebis ayant des taux de prolificité de I,5 à 2,0 (MARKov, 1952).

Ffremov (1958), envisageant l'amélioration des races locales de la province d'Udmurt par croisement avec des béliers Romanov, Tsigai, Précoce et Altai, observe chez les femelles de première génération des taux de fécondité (nombre d'agneaux nés par brebis en lutte) respectivement de $1,248-0,902-0,868-0,72 \mathrm{r}$.

\section{CONCLUSION}

Cette revue bibliographique aussi exhaustive que possible, met en évidence les potentialités de la race ovine Romanov au point de vue des caractères de reproduction; compte tenu d'une 
prolificité exceptionnelle et d'une saison sexuelle très étendue, elle semble susceptible de produire en moyenne 4 à 6 agneaux par brebis et par an dans un système intensif d'exploitation. La seule race présentant une productivité aussi élevée est la Finnoise. Encore peut-on se demander si cette dernière n'a pas été obtenue, soit par croisement entre le Romanov et différentes races britaniques, soit à partir d'une même population d'origine d'où dériveraient toutes les races nordiques à queue courte; si elle possède un format plus important et des qualités lainières supérieures, elle accuse beaucoup de ressemblances avec le Romanov : prolificité élevée, conformation médiocre et coloration similaire pour près de ro p. roo des agneaux Finnois qui naissent noirs avec une liste blanche en tête et l'extrémité des membres et de la queue blanche.

Si peu de races ont fait l'objet d'un tel nombre d'études et d'expérimentations, le Romanov nous semble cependant devoir être exploré de façon plus complète.

L'étude de ses caractéristiques sanguines devrait permettre de préciser son origine et son apparentement éventuel avec diverses races ou populations.

Sur le plan scientifique, l'étude comparée des paramètres physiologiques du Romanov et des races à prolificité moyenne est susceptible d'apporter une connaissance plus précise des processus de la reproduction, donc de leur maîtrise. De même, une analyse des paramètres génétiques de la reproduction, plus particulièrement du déterminisme héréditaire du taux d'ovulation et de la taille de la portée, permettrait de concevoir des schémas de sélection plus efficaces pour l'ensemble des races ovines. Enfin, elle constitue un matériel expérimental de premier choix pour les nutritionnistes de l'espèce ovine du fait des problèmes que pose l'alimentation rationnelle de brebis aussi productives.

Sur le plan zootechnique, il conviendrait d'étudier de façon plus rigoureuse l'absence d'anœestrus saisonnier et d'anosstrus de lactation ainsi que les conséquences d'un rythme intensif de reproduction sur les performances et la longévité des brebis. Par ailleurs, il serait intéressant de voir s'il est possible d'améliorer la conformation et les qualités bouchères du Romanov par sélection sans abaisser son niveau de production.

Dans l'immédiat, si l'on s'en réfère aux différents résultats de croisements que nous avons rapportés, nous pensons que le Romanov est à même de jouer un rôle important pour l'amélioration de la productivité de notre cheptel ovin. Une enquête réalisée par le Ministère de l'Agriculture montre que Ioo brebis donnent annuellement ri2,5 agneaux dont IoI,6 parviennent jusqu'au sevrage; ce médiocre bilan nous conduit à un déficit en viande ovine sans cesse accru par l'augmentation de notre consommation nationale. Or une expérience entreprise depuis 1964 par la Recherche agronomique montre que des demi-sang Romanov sèvrent en moyenne I,82 agneaux par brebis exploitée dans le système traditionnel d'un agnelage annuel. Aussi préconisons-nous la mise en place d'un système de croisement à double étage utilisant au premier stade des reproducteurs Romanov ou Finnois, et au deuxième stade les béliers de nos meilleures races bouchères.

Reçu pour publication en février 1971.

\section{SUMMARY}

THE ROMANOV BREED OF SHEEP

It is intended to improve the productivity of breeds of sheep by selection of pure breeds and by techniques of crossing with breeds renowned for their high level of prolificacy. The Romanov breed, which has been referred to in numerous technical and scientific publications, originates from the U. R. S. S., and is classed in the latter group.

The present bibliographical review demonstrates the importance of the Romanov breed in its country of origin, and indicates its characteristics of reproduction, growth and slaughter value. This has been the occasion to give a report on the various crossing experiments that have been carried out with this breed to improve the prolificity of other breeds.

The Romanov breed is characterized by an early sexual maturity, a high prolificity and a long sexual season. It has been used successfully for improving the productivity of other breeds. 


\section{RÉFÉRENCES BIBLIOGRAPHIQUES}

Bounkine S., Liadsky B., Sobolj $\quad$ S., Feigine A., 1934. L'élevage ovin en U. R. S. S. et les besoins de l'industrie lainière. Probl. Zivotn., 1, 129-136.

Devine K. P., Kandalov V. I., Nasonovsky N. M., Ig63. Méthodes de castration des agneaux Romanov. Ovtsevodstvo, $9(8)$, II-I2.

Dobrotvorskaia Z. L., I965. Utilisation optimum de la maturité précoce des brebis Romanov. Ovtsevodstio, 11 (II), I8-I9.

Dukine J. P., ig66. L’âge optimum pour tester les béliers sur les descendances, Ovtsevodstvo, 12 (7), $23-24$.

Efremov S. A., I958. Perspectives de développement de l'élevage ovin dans la province d'Udmurt. Zivotnovodstio, 20 (2), 13-19.

Eremeeva K. M., Starkova K. P., I960. Principes réglant le développement de la peau et de la laine chez les moutons Romanov. Trud. mosk. vet. Akad., 28, I5I-163.

Golovina V. Ia., I936. Croisements de moutons "nordique à queue courte » avec béliers Romanov. Probl. Zivotn., 9, 87-95.

Gorb T., I945. Amélioration de la situation économique des fermes d'état élevant des moutons Romanov. Sovkoz. Proizvod, 4-5, 30-33.

Guerassimov L. M., I957. Quelques particularités de reproduction des brebis Romanov. Ovtsevodstvo, $3(8), 2 I-23$.

Heptner R. A., I940. Problèmes concernant l'amélioration de la productivité en race Romanov. Sovetsk. Zootech., 2-3, 75-84.

Heptner R. A., I94I. Base scientifique de l'évaluation du mouton Romanov. Nauc. Cte. vsesojuz. Nauc. Issled. Zivotn. Rab. vypol. God. velik. Ctecut., 43, I6-I82.

IAdrilev V. I., Stepanova S. A., I965. Les agneaux Romanov peuvent être abattus avec profit à l'âge de 7-8 mois. Ovtsevodstvo, il (5), 26-27.

Kovnerev I. P., I955. Essai d'élévation de la vitalité des moutons de race Romanov. Trud. Pushkinskoi Nauchno issled., Lab. Razved Selskov. Zhivotn., 7, 6r-69.

Kovnerev I. P., I958. Élevage d'agneaux Romanov en climat froid. Ovtsevodstvo, 4 (II), $28-29$.

Kovnerev I. P., I963. Accroissement des naissances multiples chez les brebis Romanov, source d'augmentation de la production de mouton. Zivotnovodstvo, 25 (7), 50-54.

Kovnerev I. P., Mosiaguina G. A., I963. Par quelle méthode castrer les agneaux Romanov. Ovtsevodstvo, 9 (II), I6.

Kovnerev I. P., 1965. Finition intensive des Romanov. Zivotnovodstvo, 27 (I), I2-I7.

Kovnerev I. P., I966. Sélection de la race Romanov dans les Sovkhozes et les Kolkhozes. Vsesojuz. nauch.-issledovat. Ins.t Zhivotnovod., Trudy S.S.S.R., 29, 239-247.

Kovneriev I. P., Selianine G. I., ig6r. Qualité des moutons Romanov élevés dans la province de Sverdlosk pour les vêtements de peau. Ovtsevodstvo, 7 (9), 32-34.

Kovnerev I. P., Zamorychev A. V., Selianine G. I., Smirnov L. F., Iadrilev V. I., I967. Organisation et technique d'élevage des ovins Romanov, Kolos éd., Moscou, 232 pp.

Lebedev A., 1958. Chronique des films nouveaux sur les brebis. Ovtsevodstvo, 3, 46-47.

Louzina E., I943. Les moutons Romanov dans les fermes d'état. Sovkoz. Proizvod., 6/7, 26-28.

MARkov I. S., I952. Développement de l'élevage ovin dans la République des Komis. Socialist. Zivotn, $14(8), 57-59$.

Mекнтіev M. R., I966. Caractéristiques comparées de la croissance du foie chez les embryons des brebis Romanov en fonction de leur prolificité. Vsesojuz. nauch.-issledovat. inst. Zhivotnovod. Trudy S. S. S. R., 29, $248-255$.

Melekine R. P., Arzoumaniane E. E., I965. Évaluation du poids du squelette et du poids de muscle sur agneaux Romanov vivants. Zivotnovodstvo, 27 (5), 87-88.

Mojaeva E., I965. La sélection des moutons de race Romanov. Vest. Selsk. Nauki., 4, I5I-I52.

Nikolsky N. F., I933. Fertilité du Karakul Trudy Inst. Gibrid. Askania Nova, 1, 25 I-267.

PANine A. I., 1957. Bases biologiques de l'élevage des moutons Romanov. Ovtsevodstvo, 3 (Io), 26-30.

Peregone I. L., I950. Résultats d'une expérience destinée à obtenir un mouton Karakul prolifique. Karakulevodstvo 1 Zverovodstvo, 3, 25-34.

Peregone I. L., I953. Expériences de sélection d'un nouveau type de mouton à fourrure. Trud. vsesojuz nauc-iddled. Gibrid. Akklim Zivoln. Askania-Nova. Akad. Ivanova, 5, 5 I-75.

Plakhov I802 cité par Kovnerev et al., I967.

Polikarpova E. F., I964. Corrélation entre la durée de l'œstrus et les naissances multiples chez les brebis Romanov. In Zokonomernosti individualjn. razvitija sel.hoz zinotnyh. 
Polikarpova E. F., Zelensky G. G., Nevzgodina M. V., ig63. Efficacité de saillies multiples au cours d'un même costrus chez les brebis Romanov. Ovtsevodstvo, 9 (Io), Io-II.

Pokrovskaia V. A., I957. Augmentation de la productivité des brebis Romanov. Ovtsevodstvo, 3 (8), 23-25.

Selianine G. I., I957. Problèmes actuels de l'élevage des brebis Romanov. Ovtsezodstvo, 3 (7), 6-9.

Selianine G. I., I958. Effet de l'âge des brebis Romanov sur leur fertilité, et sur la valeur de leur descendance. Vestn. sel.-hoz. Nauk. Vashinil., 3 (9), 65-7o.

Selianine G. I., rg6o. Élevage de moutons Romanov dans les districts Ouraliens et trans-Ouraliens. Outsevodstvo, $6(9), 24-27$.

Selianine G. I., I965. Maturité biologique et exploitation économique des brebis Romanov. Ostsevodstvo, 11 (7), 27-29.

Selianine G. I., Koulikov V. G., I964. Qualité de boucherie des agneaux Romanot. Ontsevodstvo, $10(8), 23-25$.

Sementschenko S. P., r952. Arbeitserfahrungen in der Romanov-Schafzucht. Verlag Kultur und Fortschrit, Berlin, 80 pp.

Shoubine A. E., Zamorychev A. V., 1959. Les saisons d'agnelage des brebis Romanov. Ovtsevodstvo, $5(8), 26-28$.

Smirnov L. F., I935. Prolificité des moutons Romanov. Probl. Zivotn., 8, 7-19.

Smirnov L. F., Golovina V. Ia., I94I. Travail de sélection et d'élevage en race Romanov. Trud. Iaroslavsk. Oblast. opyt. Stanc. Zivotn., $79-92$.

Smirnov L. F., Sveco A. V., r94I. Expérience de maintien de femelles Romanov en cases individuelles comme moyen de contrôler la mortalité des agneaux. Trud. Iaroslavsk., Oblast. opyt. Stanc. Zivotn., I02-I09.

Smirnov L. F., Smirnova V. Ia, Voskoboinikov G. N., I954. I,'agnelage d'automne des brebis Romanov comme moyen d'accroître la vitalité des agneaux. Zivotnovodstvo, 10, II5-II7.

Smirnova V. Ia., I958. Production laitière des brebis Romanov. Trud. Vop. Kormodobyr. Svinovod. Ovtsevod., 3, 27I-339.

SoKolov 1853 cité par KovNeRev et al., 1967.

Starovoltenko V., Eline G. Ja., 1965. Élevage des béliers provenant de mères à portées nombreuses. Ovtsevodstvo, 11 (5), I4-15.

Stroukov r943. La coopérative "Gosplemrassadnik" d'élevage de race Ronanoz. Socialist. seljak. hoz, 10-11, 34-43.

Szymski J., I954. La race ovine Romanov d'U. R. S. S. peut améliorer la race polonajse des bruyères (Wrzosowka). Przegl. hodowl., 22 (I), 23-28.

Ussakova S. A., Fudelj T, N., I94I. Agnelages rapprochés en race Romanov. Trud. Iaroslavsk oblast. stauc. Zivotn., 93-гог.

Vorobiev P. A., I966. Origine et situation actuelle de la race Romanov. ['sesojuz. nauch,-issledovat. Inst. Zhivotnovod. Trudy S. S. S. R., 19, $232-238$.

Youdine V. M., Petrov A. V., i957. Problèmes de l'évolution de l'élevage des brebis Romanov. Ovtsevodstvo, $3(7)$, IO-I 4 .

Zamorychev A. V., I963. La production de viande d'agneaux Romanov et d'agneaux croisés. Trud Vologd. moloc. Inst., 46, 3-13.

Zeiensky G. G., Troussova T. A., I958, Engraissement des Romanov. Ottsetodstzo, 4 (5), 25. 